\title{
Review of: "Recombinantly Expressed MeICT, a new Toxin from Mesobuthus Eupeus Scorpion, Inhibits Glioma Cell Proliferation and Downregulates Annexin A2 and FOXM1 Genes"
}

\section{Fatemeh Kazemi-Lomedasht}

Potential competing interests: The author(s) declared that no potential competing interests exist.

The study is relatively well designed and executed and the results are of potential interest for the readership of the journal.

However, some major revisions should be undertaken prior to the publication of this manuscript.

Firstly, there are some issues with the English (syntax, grammar, etc.).

Introduction:

The authors do not cite relevant literature and thus seem to lack knowledge on prior art/state-of-the-art.

The methods are not sufficiently well-described for the study to be reproducible.

Discussion:

Many of the discussion sentences are redundant to the results. In fact, this section is extremely poor. Here, should be addressed the main results and problems of the study. I recommend writing a new discussion and not improve this. 